\section{GLOSSARY OF VIRTUAL REALITY TERMINOLOGY}

\author{
Carol Manetta \\ Ford Motor Credit Company \\ Dearborn, Michigan \\ Richard A. Blade \\ University of Colorado - Colorado Springs \\ Editor: Mary Lou Padgett
}

\section{ABSTRACT}

The authors place the terms and definitions contained in this arlicle into the public domain in order to elicit comments and discussion from readers. After much discussion and many modifications on the part of the Working Group on the VR Glossary, the revised glossary will be submitted as a IEEE draft standard. Then a VR Glossary Balloting Committee will be formed and ultimately vote on the inclusion of individual words and definitions into the IEEE Standard 100 Dictionary of Electrical and Electronic Terms.

\section{Introduction}

The following terms and definitions from numerous sources are being proposed to the IEEE Standards Working Group on Virtual Reality Terminology. The definitions are being placed in the public domain for discussion only and do not constitute a draft standard. The authors invite suggestions from interested persons. To suggest modifications or propose new terms and/or definitions, you may email r.blade@ieee.org or contact Richard Blade through the IJVR. If you wish to actively participate in the glossary working group, or any other standards working group, you should fill out the survey form found on the last page and send a xerox copy to Richard Blade at the IJVR.

\section{Glossary of Terms}

absolute values: Position and orientation within a virtual space as measured from a single, constant point of origin. If a real or virtual object is moved, its previous coordinates are ignored, and new position and orientation measurements are taken.

accelerator: Specialized hardware that increases the speed of graphics calculations.

accommodation: Change in the focal length of the eye's lens to maintain focus on a moving close object.

actuator: Usually mochanical (hydraulic) or electric means used to provide force or tactile feedback to a user. ambient light: Naturally occurring illumination arising from outside the apparatus.

articulation: Objects composed of several parts that are separately moveable.

artificial reality: Simulated spaces created from a combination of computer and video systems.

aspect ratio: Ratio of width to height of the field of view.

assistive agents: Artificial intelligence algorithms developed to guide users through a VR world, and to coach the user on available choices within the world

augmented reality: The use of transparent glasses on which a computer displays data so that the viewer can simultaneously view computer generated and real world scenes.

autonomy: Performance or action of the object on the rule of physics, biology, or a virtual world, but not by independent decision of a human operator.

avatar: A user's graphical persona inside a virtual world.

back clipping plane: A distance beyond which objects are not shown.

backdrop: The stationary background in a virtual world. The boundary of the world which cannot be moved or broken into smaller elements.

backface removal: The elimination of those portions of a displayed object that are facing away from the viewer.

binocular: Displaying a different image to each eye for the purpose of stereographic viewing.

binocular parallax: See parallax and motion parallax.

bi-ocular: Displaying the same image separately to each eye. Sometimes done in binocular displays to conserve computing resources when depth perception is not critical.

biosensors: Sensor devices that monitor the body's electrical activity for the purpose of computer input.

Binocular Omni-Orientation Monitor (BOOM): A 3-D display device suspended from a weighted boom that can swivel freely so the viewer can use the device by bringing the device up to the eyes and viewing the 3-D environment while holding it. The boom's position and orientation communicates the user's point of view to the computer.

browser: Overviews, such as indexes, lists or animated maps, to provide a means of navigating through the physical, temporal, and conceptual elements of a virtual world.

CAVE: VR world projected on the walls and ceiling of a room to give the illusion of immersion.

cue conflict: A theory to explain a kind of motion sickness caused when the body wies to interpret conflicting clues being received by different senses. Frequent causes are faulty calibration of eye devices or delay between the sensory inputs and output display.

computer graphics: (1) The branch of computer science concerned with methods of creating, modifying, or analyzing pictorial data. (2) The use of a computer in any discipline to create, modify, or analyze images. 
concept map: Terms, definitions, or icons arranged in semantic proximity.

convergence: Occurs in stereoscopic viewing when the left and right eye images become fused into a single image.

convolve: To filter and intertwine signals (e.g., sounds) and render them three-dimensional. Used in VR applications to recreate sounds that give directional cues.

Convolvotron: An output system for controlling binaural sound production in a VR world.

coordinates: A set of data values that determine the location of a point in a space. The number of coordinates corresponds to the dimensionality of the space.

culling: Removing invisible pieces of geometry and only sending potentially visible geometry to the graphics subsystem. Simple culling rejects entire objects not in the view. More complex systems take into account occlusion of some objects by others, e.g. a building hiding trees behind it.

cyberspace: A computer synthesized reality. Often a computer synthesized 3-D space. See also: virtual reality.

cyborg: A robotic humanoid modeled diroctly from digital readings of a real human and transformed into a photo realistic, animated character produced via illusionary metamorphosis.

data sonification: Assignment of sounds to digitized data which may involve filtering to give illusion of localized sound.

data spacialization: Assignment of orientation (yaw, pitch) and position coordinates $(x, y, z)$ to digital sounds assigned to data.

DataGlove: A glove wired with sensors and connected to a computer system for gesture recognition and navigation through a virtual environment. Known generically as a "wired glove".

Deformable Object Technology (DOT): Virtual objects which bend and deform appropriately when touched.

depth cueing: Use of shading, texture, color, interposition, or other visual characteristics to provide a cue for the distance of an object from the observer.

dolly shot: Display of a scene while moving forward or backward. (See also pan shot and track shot.)

Doppler effect: An apparent increase in the frequency of sound or light as its source approaches an observer or a decrease if it moves away.

dynamic lighting: Changes in lighting effects as objects or the observer move.

dynamics: The rules that govern all actions and behaviors within the environment.

effectors: Interfacing devices used in virtual environments for input/output, tactile sensation and tracking. Examples are gloves, head mounted displays, headphones, and trackers. egocenter: The sense of one's own location in a virtual environment.

environment: In VR terms, this is a computer-generated model that can be experienced by an observer as if it were a place.

exoskeleton: mechanically linked structure for control of and feedback from an application. \{\{photo\}\}

eye clearance: The most accurate figure of merit used to describe the HMD positioning relative to the eye.

eye tracking: Measurement of the direction of gaze.

eyeball in the hand: A metaphor for visualized tracking where the tracker is held in the hand and is connected to motion of the projection point of the display.

field of view (FOV): The angle in degrees of the visual field. Since a human's two eyes have overlapping 140 degree FOV, binocular or total FOV is roughly 180 degrees in most people. A feeling of immersion arises with FOV greater than roughly 60 to 90 degrees.

fish tank VR: The egocenter of an observer looking "through" a computer monitor to a virtual outside world using a stereoscopic display system. That is, to a person looking through a stereo "window" to a virtual "outside", the person imagines him/herself to be in a fish tank.

force feedback: An output device that transmits pressure, force or vibration to provide the VR participant with the sense of resisting force, typically to weight or inertia. This is in contrast to tactile feedback, which simulates sensation applied to the skin.

fractal: A self-similar graphical pattern generated by using the same rules at variou's levels of detail. That is, a graphical pattern that repeats itself on a smaller and smaller scale.

frustum of vision: Three-dimensional field of view in which all modeled objects are visible.

gesture: Hand motion that can be interpreted as a sign, signal, or symbol.

Gouraud shading: The shading of polygons smoothly with bilinear interpolation.

haptic interfaces: Use of physical sensors to provide users with a sense of touch at the skin level, and force foedback information from muscles and joints.

\{\{photo 1\}\}

head tracking: Monitoring the position and orientation of the head through various tracking devices.

head-coupled: Displays or robotic actions that are activated by head motion through a head racking device.

head-related transfer function: A mathematical transformation of sound spectrum that modifies the amplitude and phase of acoustic signals to take into account the shape effects of the listener's head.

heads-up display: A display device that allows users see graphics superimposed on their view of the real world.

hidden surface: A surface of a graphics object that is occluded from view by intervening objects. 
head mounted display (HMD): A set of goggles or a helmet with tiny monitors in front of each eye to generate images seen by the wearer as threedimensional. Often the HMD is combined with a head tracker so that the images displayed in the HMD changes as the head moves. \{\{photos\}\}

image distance: Perceived distance to the object. (In contrast to the real object distance, if there exists a real object.)

immersion: The observer's emotional reaction to the virtual world as being part of it.

interaural amplitude: Differences between a person's two ears in the intensity of a sound, typically due to the location of the sound.

interaural time: Differences between a person's two ears in the phase of a sound, typically due to the location of the sound.

interface: A set of devices, software, and techniques that connect computers with people to perform tasks.

Internet: A world wide digital network capable of supporting shared virtual worlds.

inverse kinematics: A specification of the motion of dynamic systems from properties of their joints and extensions.

joystick: An input device that consists of a short lever gripped with one hand to be moved from side to side or towards and away from the person. Frequently it is used to navigate in a virtual space.

kinesthesis: Sensations derived from muscles, tendons and joints and stimulated by movement and tension.

kinesthetic dissonance: Mismatch between feedback or its absence from touch or motion during VR experiences.

latency: Lag between user motion and tracker system response, sometimes measured in frames. Delay between actual change in position and reflection by the program. Delayed response time.

Liquid Crystal Display (LCD): Display devices that use bipolar films sandwiched between thin panes of glass. They are lightweight and ransmissive or reflective, and are often used in HMDs.

level of detail (LOD): A model of a particular resolution among a series of models of the same object. Greater graphic performance can be obtained by using a lower LOD when the object occupies fewer pixels on the screen or is not in a region of significant interest.

magic wand: A three-dimensional input device used for pointing and interaction. A kind of three-dimensional mouse.

metaball: A surface defined about a point specified by a location, a radius, and an "intensity". When two metaballs come in contact, their shapes blend together.

metallic distortion: Noise interference or degraded performance in electromagnetic trackers when used near large metallic objocts.

model: A computer-generated simulation of something real. motion parallax: A means whereby the eyes can judge distance by noticing how closer objects appear to move faster than distant ones.

motion platform: A controlled physical system that provides real motion to simulate the displayed motion in a VR world. \{\{photo\}\}

navigation: Purposeful motion through virtual space.

objects: Discrete 3-D shapes within the virtual world that a user can interact with.

occipital cortex: The back of the brain receiving retinotopic projections of visual displays.

occlusion: Hiding an object or a portion of an object from sight by interposition of other objects.

pan: The angular displacement of a view along any axis or direction in a three-dimensional world.

pan shot: Display of a scene while moving about any axis. (See also dolly shot and track shot.)

parallax: The difference in viewing angle created by having two eyes looking at the same scene from slightly different positions, thereby creating a sense of depth. (Also referred to as binocular parallax.)

parietal cortex: An area of the brain adjacent and above the occipital cortex, thought to process spatial location and direction information.

perspective: The rules that determine the relative size of objects on a flat viewing surface to give the perception of depth.

phong shading: A method for calculating the brightwess of a surface pixel by linearly interpolating points on a polygon and using the cosine of the viewing angle. Produces realistic shading.

photo realism: An attempt to create realistic appearing images with great detail and texture.

pitch: The angular displacement of the lateral axis about a horizontal axis perpendicular to the lateral axis.

pixel: The smallest element of a display that can be adjusted in intensity.

platform VR: Individual or shared VR displays built into physical mockups of vehicles and other physical settings.

polygon: A display element that consists of an area enclosed by a set of broken straight lines.

portal: Polygons or icon that a user can pass through in a virtual space to automatically load a new world or execute a user-defined function. A three-dimensional version of an interactive icon in multimedia.

position sensor: A tracking device that provides information about its location and/or orientation.

position trigger: A hotspot, sensitive spot, or button that causes a change in the computer program when touched in some way.

presence: A feeling of being immersed in an environment, able to interact with objects there. A defining characteristic of a VR system. 
radiosity: A diffuse illumination calculation system for graphics based on energy balancing that takes into account multiple reflections off many walls.

ray tracing: A technique for displaying a threedimensional object with shading and shadows by tracing light rays backward from the viewing position to the light source.

real time: Action taking place with no perceptible or significant delay after the input that initiates the action.

real-time imaging: Graphics or images synchronized with real-world time and events.

reality engine: A computer system for generating virtual objects and environments in response to user input, usually in real time.

refresh rate: The frequency with which an image is regenerated on a display surface.

resolution: Usually the number of or pixels in a VR display.

retinal binocular disparity (RBD): Ratio of the convergence angle of the image to the convergence angle of the object.

roll: Angular displacement about the lateral axis.

scene view: Virtual display viewed on a large screen or through a terminal window rather than with immersive devices.

semiocclusion: Occlusion to one eye only.

shared worlds: Virtual environments that are shared by multiple participants.

shutter glasses: Glasses that alternately block out the left and right eye views in synchrony with the computer display of left and right eye images to provide stereoscopic images on the computer screen. \{\{photo\}\}

simulator sickness: Various disturbances, ranging in degree from a feeling of unpleasantness, disorientation, and headaches to extreme nausea, caused by various aspects of a simulator. Possible factors include sensory distortions such as abnormal movement of arms and heads because of the weight of equipment; long delays or lags in feedback, and missing visual cues from convergence and accommodation.

six degrees of freedom (6DOF): Ability to move in three spatial directions and orient about three axes passing through the center of the body. Thus the location and orientation are specified by six coordinates.

spatial navigation: Self orientation and locomotion in virtual worlds.

stereopsis: Binocular vision of images with different views by the two eyes to distinguish depth.

tactile displays: Devices that provide tactile and kinesthetic sensations.

Techsplanation: Use of VR technology and other communication technology to explain or teach. tele-existence: Virtual reality experienced from remote locations. ${ }^{1}$

telemanipulation: Robotic control of distant objects.

TELEOS $^{\text {m}}$ : A tool to create Silicon Graphics computerbased real-time interactive environments with 'life-like' deformable objects. ${ }^{2}$

teleoperator: Person doing telemanipulation.

telepresence: Remote control with adequate sensory data to give the illusion of being at that remote location.

temporal lobe: An area of the brain in front of the occipital cortex and the parietal cortex which is the receiving site for hearing.

terrain: Geographical information and models that can be either randomly generated or based on actual data.

texture mapping: A bitmap pattern added to an object to increase realism.

three-dimensional graphics: The presentation of data on a two-dimensional display surface so that it appears to represent a three dimensional model.

track shot: Rotating display of the same scene. (See also dolly shot and pan shot.)

tracker: A device that provides numeric coordinates to identify the current position and/or orientation of an object or user in real space.

universe: The collection of all entities and the space they are embedded in for a VR world.

viewpoints: Points from which raytracing and geometry creation occurs. The geometric eye point of the simulation.

vection: Sensation of egocenter caused by motion of the visual environment.

virtual environments: Realistic simulations of interactive scenes.

Virtual MIS: Use of computer models and specialized interaction devices that mimic surgical tools to allow medical personnel to practice Minimally Invasive Surgery procedures.

virtual prototype: Simulation of an intended design or product to illustrate the characteristics before actual construction. Usually used as an exploratory tool for developers or as a communications prop for persons reviewing proposed designs.

virtual reality: A computer system used to create an artificial world in which the user has the impression of being in that world with the ability to navigate through the world and manipulate objects in the world.

virtual world: Whole virtual environment or universe within a given simulation.

${ }^{1}$ Input from one individual indicates that tele-existence is an English translation of the term used in Japan for telepresence.

2 Some persons have objected to the inclusion of a trademarked commercial product name. In the past, however, other such names, such as the Boom and the Data Glove, have become generic terms. This term was submitted by the holder of the trademark, and we leave the issue of inclusion to the balloting committee. 
visualization: The ability to graphically represent abstract data that would normally appear as text and numbers on a computer.

voxel: A cubic volume pixel for quantizing threedimensional space.

world in the hand: A metaphor for visualized tracking where a tracker is held in the hand and is connected to the motion of an object in a display.

yaw: The angular displacement about the vertical axis.

\section{ACKNOWLEDGMENTS}

The authors wish to acknowledge the input of the many persons who have provided suggestions regarding this glossary. Of particular value has been the extensive input from Shojiro Nagata of the Human Factors Division, NHK Science and Technical Research Labs, Tokyo (nagata@strl.nhk.or.jp).

We would also like to acknowledge the usefulness of the Sci.Virtual-Worlds newsgroup on the Internet. It provided us with an effective means of getting the terms out to the general computing public.

\section{REFERENCES}

The authors began with terms and definitions from various books and presentations, including the following:

Begault, D., 3-D Sound for Virtual Reality and Multimedia, Academic Press, 1994.

Benedikt, M. (ed.), Cyberspace: First Steps, MT Press, 1991.

Bryson, S., "Direct Manipulation in Virtual Reality", Tutorial proceedings, VRAIS '95, 1995.

Eamshaw, R. A., Gigante, M. A., Jones, H., Virtual Reality Systems, Academic Press.

Ellis, S. R. (ed.), Pictorial Communication in Virtual and Real Environments, Taylor and Francis, 1991.

Kalawsky, R., The Science of Virtual Reality and Virtual Environments, Addison-Wesley, 1993.

Latham, R., The Dictionary of Computer Graphics Technology and Applications, Springer-Verlag, 1991.

Laurel, B., Computers as Theater, Addison-Wesley, 1991.

Pimentel and Teixeira, Virtual Reality; through the new looking glass, Intel, 1992.

Rheingold, H., Virtual Reality, Simon \& Schuster, 1991.

Rolland, J., "Fundamentals of Optics in Head Mounted Displays", Tutorial proceedings, VRAIS '95, 1995.
Sowizral, H., "An Introduction to Virtual Reality", Tutorial proceedings, VRAIS '95, 1995.

Wexelblat, A. (ed.), Virtual Reality Applications and Explorations, Academic Press, 1993.

At the point of this publication, input from various individuals has resulted in significant changes in the definitions.

\section{BIOGRAPHIES}

CAROL MANETTA is an Instructional Designer, Ford Motor Credit Company, Dearborn, Michigan, where she is currently developing multimedia courses. She holds a masters degree in Instructional Technology from Wayne State University. She has researched virtual reality in the United States and Japan. Her other professional associations include the American Society for Training and Development, and the National Society for Performance and Instruction. She serves as Vice Chair of the IEEE Working Group on the VR Glossary.

\section{Contact information:}

Carol Manetta

Phone: 313-845-1263

FAX: $313-337-2721$

Email: cmanetta@aol.com

RICHARD BLADE \{\{photo $\}\}$ is a Professor of Physics at the University of Colorado - Colorado Springs. Other professional positions include Caltech, University of California - Berkeley, and United States International University. Professor Blade serves as science editor for IPI Press and Editor-in-Chief of the IJVR. He also serves as Focus Chair for IEEE Standards in Virtual Reality and Chair of the Working Group on the VR Glossary. His current research involves VR motion simulation.

\section{Contact information:}

Richard A. Blade

IPI Press

2608 N. Cascade Ave.

Colorado Springs, CO 80907

Pers Phone: 719-471-4476

FAX: 719-630-1427

Email: rblade@mail.uccs.edu 